\title{
Neoadjuvant cryotherapy improves dysphagia and may impact remission rates in advanced esophageal cancer
}

\section{다 (1) $\odot$}

Authors

Tilak Shah 1,2,3, Vladimir Kushnir ${ }^{4}$, Pritesh Mutha', ${ }^{1,2}$ Mankanchan Majhail ${ }^{1}$, Bhaumik Patel ${ }^{3,5}$, Matthew Schutzer ${ }^{6}$, Drew Mogahanaki ${ }^{6,3}$, George Smallfield², Milan Patel ${ }^{7}$, Alvin Zfass ${ }^{1,2}$

Institutions

1 Division of Gastroenterology, Hunter Holmes McGuire VA Medical Center, Richmond, Virginia, United States

2 Division of Gastroenterology, Virginia Commonwealth University Health System

3 Massey Cancer Center, Virginia Commonwealth University, Richmond, Virginia, United States

4 Section of Gastroenterology, Washington University in St. Louis, St. Louis, Missouri, United States

5 Division of Hematology-Oncology, Hunter Holmes McGuire VA Medical Center, Richmond, Virginia, United States

6 Radiation oncology Service, Hunter Holmes McGuire VA Medical Center, Richmond, Virginia, United States

7 Department of Internal Medicine, Virginia Commonwealth University, Richmond, Virginia, United States

submitted 25.1.2019

accepted after revision 25.4.2019

\section{Bibliography}

DOI https://doi.org/10.1055/a-0957-2798 |

Endoscopy International Open 2019; 07: E1522-E1527

(c) Georg Thieme Verlag KG Stuttgart · New York eISSN 2196-9736

Corresponding author

Tilak Shah, MD, MHS, McGuire VAMC, Gastroenterology; 111 N, 1201 Broad Rock Blvd, Richmond, VA 23224

Fax: +1-804-675-5816

Tilak.Shah@va.gov

\section{ABSTRACT}

Background and study aims Liquid nitrogen spray cryotherapy (LNSC) can provide rapid dysphagia relief, and is postulated to stimulate a local antitumor immune response. The aim of this prospective pilot clinical trial was to evaluate the safety and efficacy of LNSC when administered prior to chemoradiotherapy.

Patients and methods Treatment-naïve adult patients with dysphagia at the time of biopsy-proven squamous carcinoma or adenocarcinoma of the esophagus were prospectively enrolled at two tertiary medical centers. Patients underwent a single session of LNSC. The primary outcome measure was change in dysphagia at 1 and 2 weeks postcryotherapy. A secondary outcome measure was clinical complete response rate $(C R)$ following chemoradiotherapy. Results Twenty-five patients were screened, of whom 21 patients were eligible and enrolled. There were seven with metastatic and 14 with locally advanced cancer. The primary outcome of dysphagia improvement of $\geq 1$ point occurred in $15 / 21$ patients ( $71 \%$ ) at 1 week, and $10 / 20$ patients ( $50 \%$ ) at 2 weeks. The median dysphagia score improved by 1 at 1 week $(P=0.0003)$, and 0.5 at 2 weeks $(P=0.02)$. Six of nine patients (67\%) with locally advanced cancer who completed chemoradiation did not have residual tumor cells on mucosal biopsy, and five of nine patients (56\%) had a clinical CR. There were no serious cryotherapy-related complications. Conclusions LNSC provided safe and effective palliation for esophageal cancer patients who presented with dysphagia at index diagnosis. Its combination with chemoradiotherapy did not lead to any serious toxicity. Our study provides a scientific rationale for pursuing larger clinical trials addressing synergistic effects of combining LNSC with chemoradiation.

\section{Introduction}

In medically fit patients with advanced esophageal cancer, the National Comprehensive Cancer Network (NCCN) recommends chemotherapy with or without radiation [1]. Unfortunately, despite undergoing chemoradiation and surgery, 5-year survival for patients with locally advanced esophageal cancer ranges from only $20 \%$ to $40 \%$ [2,3]. In a randomized trial of 386 pa- tients, a pathologic complete response $(C R)$ was observed in only $29 \%$ of patients who underwent neoadjuvant chemotherapy with carboplatin and paclitaxel along with concurrent radiotherapy at 41.4 Gray (Gy) [4]. Even after undergoing surgery, median survival was only 49.4 months.

Dysphagia is the most common symptom in patients with advanced esophageal cancer. It contributes to malnourishment and weight loss, and may delay initiation of chemoradiation. 
Self-expandable metal stents can rapidly palliate dysphagia, but their use in the neoadjuvant setting is associated with a high morbidity rate, increased risk of serious toxicities, and possibly decreased survival [5]. Furthermore, $50 \%$ of these stents migrate during neoadjuvant chemotherapy.

Chemoradiation does improve dysphagia, but the effects are not observed for several weeks after the initial dose of radiation is administered. Enteral feeding via gastrostomy or jejunostomy tubes can ameliorate malnutrition, but they do not palliate dysphagia. Furthermore, gastrostomy tubes can render the gastric conduit unsuitable for esophagectomy [6]; jejunostomy tubes require surgery performed under general anesthesia.

Liquid nitrogen spray cryotherapy (LNSC) is a relatively new modality for treatment of esophageal neoplasia. During LNSC, liquid nitrogen at super cold temperatures $\left(-76\right.$ to $\left.-158^{\circ} \mathrm{C}\right)$ is sprayed directly on the tumor for 20 to 40 seconds $[7,8]$. The tissue is then allowed to thaw before spraying again for 20 to 40 seconds. Typically, two to four freeze-thaw cycles of liquid nitrogen are administered. These cycles cause intracellular disruption and ischemia, which leads to ablation of tumor tissue [9]. In addition, early observations that freezing a primary lesion may cause untreated sites of disease to regress suggest that cryoablation may stimulate an antitumor immune response [9-11].

Several studies have documented the safety and effectiveness of LNSC in Barrett's esophagus and superficial esophageal cancer [12-14]. Retrospective series and case reports suggest that LNSC is also safe and efficacious in patients with advanced esophageal cancer $[5,13,15-27]$. However, no study has prospectively assessed the efficacy of LNSC in the neoadjuvant or definitive chemoradiation settings. The aims of this prospective pilot clinical trial were to evaluate: (1) safety of LNSC administered prior to chemotherapy and radiation; (2) efficacy of LNSC at palliating dysphagia at index diagnosis; and (3) efficacy of combined LNSC plus chemoradiation at inducing a clinical or pathologic complete response in locally advanced esophageal cancer.

\section{Patients and methods}

\section{Eligibility criteria}

Adult patients with dysphagia at the time of biopsy-proven squamous carcinoma or adenocarcinoma of the esophagus who were treatment-naïve were prospectively enrolled at two tertiary medical centers. One site was a tertiary veterans hospital (Hunter Holmes McGuire Veterans Affairs Medical Center) and the other was an academic medical center (Washington University Medical Center). The study was registered on clinicaltrials.gov (identifier NCT02606396). The institutional review board at both institutions approved the study. Exclusion criteria were as follows: inability to provide informed consent, absence of dysphagia, alternative etiology for dysphagia, inability to pass gastroscope and orogastric decompression tube into the stomach, and uncorrected coagulopathy (international normalized ratio $>2$ or platelet count $<50,000 / \mathrm{mm}^{3}$ ). Baseline variables recorded were as follows: age, gender, ethnicity, tumor
- Table 1 Mellow-Pinkas Dysphagia Score.

\begin{tabular}{|l|l|}
\hline Score & Symptom \\
\hline 0 & No Dysphagia \\
\hline 1 & Dysphagia to Solids \\
\hline 2 & $\begin{array}{l}\text { Dysphagia to semi-solids (grits or other foods like } \\
\text { oatmeal, cream of wheat, rice) }\end{array}$ \\
\hline 3 & Dysphagia to liquids \\
\hline 4 & Dysphagia to saliva \\
\hline
\end{tabular}

histology and TNM stage, location and length of tumor, presence or absence of critical organ invasion (i.e. aorta, trachea, heart, and vertebral body), Eastern Cooperative Oncology Group (ECOG) performance status, and baseline Mellow-Pinkas dysphagia score, a standardized and validated measure of dysphagia in patients with esophageal cancer [18] (॰ Table 1).

\section{Cryotherapy treatment}

Cryoablation was performed under monitored anesthesia either at the same time as the initial staging endoscopic ultrasound (EUS) procedure or as a separate procedure if EUS was not indicated (i.e. metastatic disease at diagnosis). Following upper endoscopy (EGD), a modified orogastric decompression tube was passed over a guidewire through the mouth into the stomach before initiation of LNSC. The tube contains decompression ports spanning the distal 12 inches to allow gas venting of both the stomach and the esophagus during treatment. The tube was connected to continuous suction throughout the cryotherapy procedure.

LNSC was performed with low-pressure liquid nitrogen (<5 psi) at $25 \mathrm{~W}$ using a cryotherapy catheter passed through the working channel of the endoscope. Two cycles of cryospray ablation of normal mucosa for 20 seconds each produces a mean depth of injury of $5.4 \mathrm{~mm}$, which is sufficient to reach the submucosa [19]. Published studies on LNSC in superficial esophageal cancer have typically reported spraying each site three times for 20 seconds during each cycle [13]. In clinical practice, a dose of 30 seconds during each cycle is more frequently used in patients with esophageal cancer [17]. Our protocol was to ablate for 20 to 40 seconds after a bed of frost was visible during each cycle ( $\triangleright$ Fig. 1). The protocol allowed for up to three cycles per site. During each cycle, a surface area of approximately 2 to $3 \mathrm{~cm}^{2}$ was targeted. Up to three sites were treated during each session. Tissue thawing was verified between cycles by waiting at least 60 seconds before retreatment, and by observing a return to baseline color of the tissue.

After the procedure was completed, patients were monitored in the recovery unit for at least 30 minutes. Patients were discharged after monitoring if they met the endoscopy unit's standard discharge criteria. All patients received standardized discharge instructions, which included clear liquids for 72 hours, avoiding hard crunchy foods for a week, and avoidance of anticoagulants and nonsteroidal anti-inflammatory 

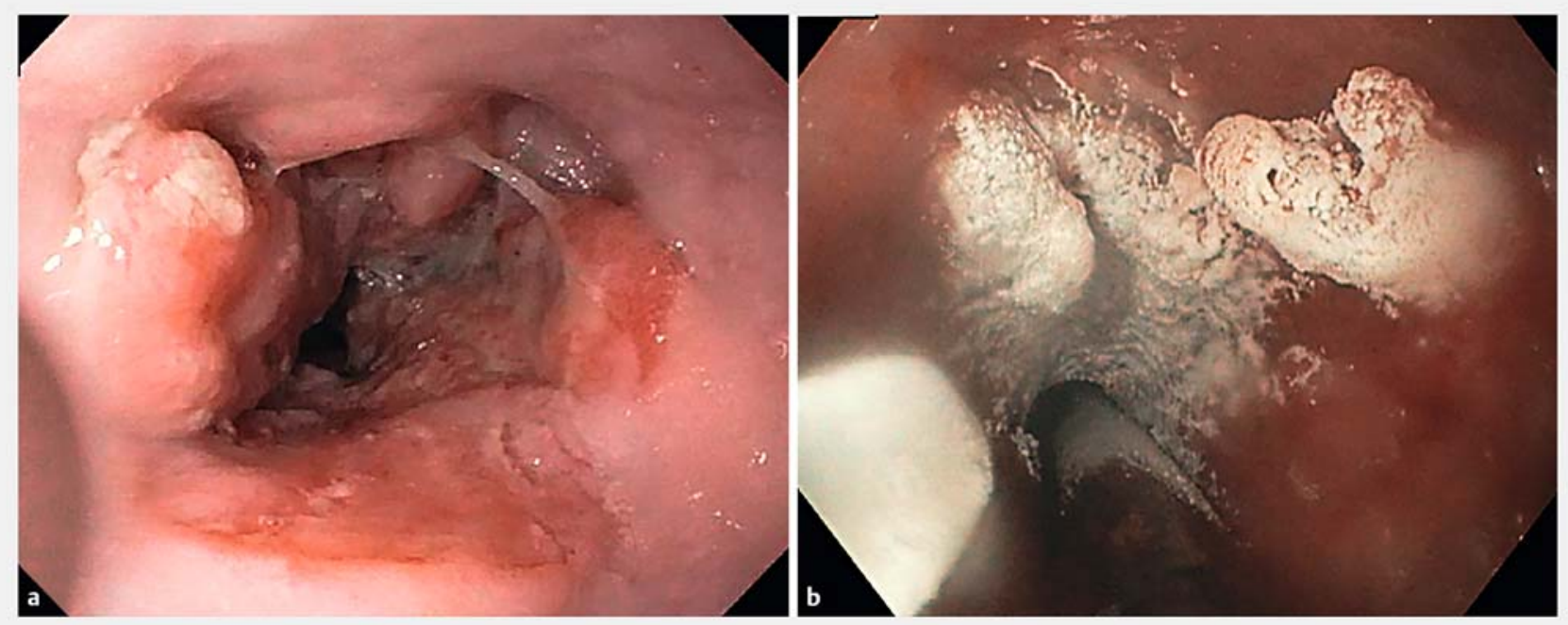

Fig. 1 a Esophageal tumor causing luminal obstruction. b Spray cryotherapy is continued for 20 to 40 seconds after a bed of frost has formed.

drugs unless the cardiopulmonary risk of discontinuing these agents was deemed unacceptable.

\section{Data collection}

A member of the study team contacted each patient 1,2 , and 4 weeks after the cryotherapy procedure to assess for procedurerelated adverse events (AEs) as outlined in the American Society of Gastrointestinal Endoscopy (ASGE) Lexicon [20] and change in Mellow Pinkas dysphagia score.

A data safety monitoring committee met every 6 months to adjudicate AEs. In patients with locally advanced disease, EGD was performed at least 6 weeks after completing chemoradiation to maximize diagnostic accuracy by minimizing the potential for sampling non-viable tumor cells [21]. The endoscopist obtained a minimum of eight mucosal biopsies to assess for presence of residual tumor cells. In addition, post-treatment positron emission tomography (PET) findings were reviewed with the multidisciplinary oncology team to assess for elevated standardized uptake values (SUV) in regional lymph nodes, distant lymph nodes, and distal organs. In patients with locally advanced cancer, data collection ended after post-chemoradiation PET scan and biopsy/surgical explant results were available. We also reviewed all patients' records to assess for recurrence and survival immediately prior to preparation of the manuscript.

\section{Statistical analyses}

The primary outcome was change in dysphagia score compared to baseline at 1 and 2 weeks after cryotherapy. The minimum clinically important difference was defined as a 1-point improvement in pre-and post-cryotherapy dysphagia score. In a series of six post-chemoradiation patients who underwent 12 sessions of cryotherapy while receiving chemotherapy, we found that dysphagia score improved in all patients, and median improvement in dysphagia score was 1.6 [22]. Absent any concurrent local or systemic therapy, we hypothesized that cryotherapy would result in a 1-point improvement in dysphagia score in at least $50 \%$ of patients. We further hypothesized that $10 \%$ of patients would have had a similar improvement in dysphagia due to dilation effect from the gastroscope and/or echoendoscope alone. Based on a confidence level of $95 \%$ and power of $80 \%$, we calculated a sample size of at least 17 patients would be needed. Therefore, we planned to enroll 20 patients for this study. Wilcoxan rank sum test was used to calculate if the change in dysphagia scores from baseline were statistically significant [23].

Secondary outcomes for the study were the proportion of patients with locally advanced disease who completed chemoradiation and had a local complete response (defined by absence of residual tumor cells on mucosal biopsy or surgical explant), and pathologic or clinical complete response (defined as absence of residual tumor cells on mucosal biopsy or surgical explant, and absence of regional or distal metastases on PET scan).

\section{Results}

A total of 25 patients were screened for inclusion in the study (CONSORT flowchart, $>$ Fig. 2). Three patients were excluded because they denied any symptoms of dysphagia. One patient was excluded after enrollment because he was found to have a superficial cancer in the setting of a large paraesophageal hernia. In this patient, the paraesophageal hernia was deemed responsible for the dysphagia symptoms. Twenty-one patients were eventually included in the study ( 14 with locally advanced cancer, and 7 with metastatic cancer). Patients were predominantly elderly, white, and male with comorbid conditions ( $\vee$ Table 2$)$. Seventeen patients $(65 \%)$ were over age 65 and five patients ( $24 \%$ ) were over age 80 at diagnosis. EUS was performed in 16 patients. In 14 of 16 , the tumor extended past the muscularis propria into the adventitia (T3 tumor), and in two of six patients the tumor extended to the muscularis propria ( $T 2$ 


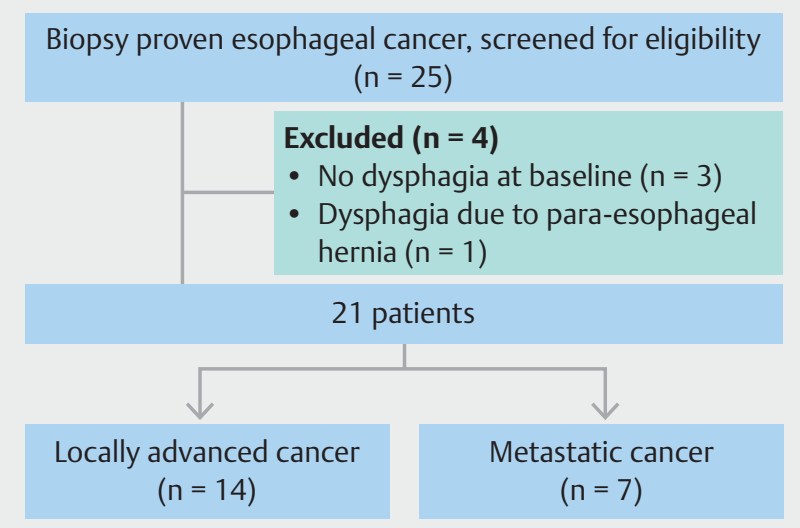

- Fig. 2 Study flowchart.

\begin{tabular}{|c|c|c|}
\hline \multirow[t]{2}{*}{ Tumor histology } & Adenocarcinoma & 15 \\
\hline & Squamous cell carcinoma & 6 \\
\hline \multirow[t]{5}{*}{ Stage at diagnosis } & Stage 1B & 1 \\
\hline & Stage 2B & 7 \\
\hline & Stage $3 \mathrm{~A}$ & 3 \\
\hline & Stage 3B & 3 \\
\hline & Stage 4 & 7 \\
\hline Median age (years) & \multicolumn{2}{|l|}{71 (range $49-87$ ) } \\
\hline Race & \multicolumn{2}{|l|}{5 black, 16 white } \\
\hline Gender & \multicolumn{2}{|l|}{20 Male; 1 Female } \\
\hline Median ASA status & \multicolumn{2}{|l|}{3 (range 1 to 4 ) } \\
\hline $\begin{array}{l}\text { Median ECOG performance } \\
\text { status }\end{array}$ & \multicolumn{2}{|l|}{1 (range 0 to 3 ) } \\
\hline Median tumor length (cm) & \multicolumn{2}{|l|}{5 (range 2 to 12 ) } \\
\hline $\begin{array}{l}\text { Mean baseline dysphagia } \\
\text { score }\end{array}$ & \multicolumn{2}{|l|}{1.76 (range 1 to 4 ) } \\
\hline Median BMI & \multicolumn{2}{|l|}{25 (range 16 to 35 ) } \\
\hline $\begin{array}{l}\text { ASA, American Society of Anes } \\
\text { Oncology Group; BMI, body ma }\end{array}$ & $\begin{array}{l}\text { logists; ECOG, Eastern Cooper } \\
\text { lex }\end{array}$ & \\
\hline
\end{tabular}

tumor). The proximal extent of the tumor was less than $30 \mathrm{~cm}$ from the incisors in 4 patients.

A median of two sites were treated during each cryotherapy session (range 1 to 3 ), and a median of four ablations were performed during each session (range 3 to 8 ). Nearly all ablations lasted 30 seconds after a bed of frost had formed. Six patients required esophageal dilation to permit passage of either the echoendoscope or the gastroscope along with the orogastric decompression tube. There were no serious AEs related to the cryotherapy procedure. One patient experienced mild chest discomfort that resolved within 20 minutes without any intervention. One patient experienced worsening dysphagia 24 hours after the procedure. An EGD was repeated, which demonstrated tumor slough obstructing the lumen. After the slough was pushed into the stomach, the patient's dysphagia improved compared to baseline. Dysphagia improvement of $\geq 1$ point occurred in 15 of 21 patients (71\%) at 1 week, and 10 of 20 patients (50\%) at 2 weeks. For the primary outcome, median dysphagia score improved by 1 at 1 week $(P=0.0003)$, and 0.5 at 2 weeks $(P=0.02)$. Dysphagia improvement of $\geq 1$ point was observed in seven of 17 patients at 4 weeks.

Among patients who did not undergo dilation, dysphagia improvement of $\geq 1$ point occurred in 11 of 15 patients (73\%) at 1 week, and nine of 14 patients (64\%) at 2 weeks. Change in dysphagia was not significantly different between patients with adenocarcinoma vs. squamous cell carcinoma $(P=1.0$ at 1 week, 0.08 at 2 weeks). Change in dysphagia was not significantly different between patients with locally advanced and metastatic cancer ( $P=0.7$ at 1 week, 0.58 at 2 weeks). One patient with metastatic disease died within 2 weeks of the procedure, and another two patients with metastatic disease died within 4 weeks. One patient with metastatic disease required stent placement. Therefore, dysphagia score was not obtained for one patient at 2 weeks, and 4 patients at 4 weeks. None of the deaths were deemed by the data safety monitoring board to be related to the cryotherapy procedure.

Among the 14 patients with locally advanced disease, treatment allocation was as follows: two patients proceeded directly to surgery without neoadjuvant chemoradiation; one patient with locally advanced disease did not receive chemotherapy because of coexisting myelodysplastic syndrome; one patient had poor performance status (ECOG 3 ) that precluded chemoradiation; one patient elected to halt chemoradiation prematurely; and nine patients completed chemoradiation. Chemoradiotherapy was initiated a median of 27 days after cryotherapy.

Of the nine patients who completed chemoradiation, only two patients required enteral feeding and no patient underwent esophageal stenting. Both patients who required enteral feeding had at least at 1-point improvement in dysphagia at 1 and 2 weeks, but had worsening dysphagia at 4 weeks after the LNSC procedure was completed. In both patients, jejunostomy tube feeding was required to maintain nutritional intake during chemoradiation. Median weight loss from diagnosis to the end of chemoradiation was $12 \mathrm{lb}$ (median total body weight loss of $5 \%$ ), and median decline in albumin was $0.4 \mathrm{~g} / \mathrm{dL}$. Median radiation dose was 50.4 Grays (range 26.6 to 66.6 Grays). The chemotherapy regimen selected for all patients with locally advanced cancer was carboplatin and paclitaxel.

A local complete response (CR) was seen in $67 \%$ of patients who completed chemoradiation, and $56 \%$ had a clinical CR. None of the patients underwent surgery after completing chemoradiation. All seven patients with metastatic cancer died during the study period. Among the patients who initiated chemoradiation, $80 \%$ patients with a clinical CR were alive but only $40 \%$ of patients who did not have CR were alive at the end of the study period. 


\section{Discussion}

In locally advanced esophageal cancer, response to induction chemoradiotherapy is the single most important predictor of prognosis [24]. Dysphagia and malnourishment are common in advanced esophageal cancer, and can impair ability to initiate and complete chemoradiation. Despite the advanced age and comorbidities of our study population, cryotherapy did not result in any serious treatment-related toxicity, and significantly improved dysphagia. Among the patients with locally advanced cancer who initiated chemoradiation, only $20 \%$ required enteral feeding; $90 \%$ of these patients were able to complete chemoradiotherapy. Total body weight loss during chemoradiation was similar to what has been described with enteral feeding and stents [25]. Improved dysphagia has also been reported with other endoscopic ablation modalities such as photodynamic therapy (PDT) and Nd:YAG laser therapy. In a randomized trial, Mellow-Pinkas dysphagia score improved by at least 1 point in less than half the patients at 1 week, and at 1 month despite multiple sessions [26]. More importantly, in contrast to LNSC, a substantial proportion of patients undergoing ablation with these modalities experienced a treatmentrelated $\mathrm{AE}$ (almost $50 \%$ of patients with PDT and $15 \%$ with $\mathrm{Nd}$ : YAG) [26]. An esophageal perforation or a pleural effusion occurred in $10 \%$ of PDT-treated patients and $8 \%$ of Nd:YAG-treated patients. While our preliminary data suggest that LNSC may be more effective and safer than these other modalities, larger studies are necessary to corroborate these findings. In our study, patients underwent a single session of LNSC. Whether multiple sessions can increase palliative efficacy of LNSC without increasing risk of AEs in the neoadjuvant setting also remains to be determined.

In the landmark Dutch CROSS study, neoadjuvant chemoradiation improved survival compared to surgery alone in locally advanced esophageal cancer [4]. In a randomized trial for locally advanced squamous cell carcinoma, neoadjuvant chemoradiation alone provided similar survival benefit compared to chemoradiation and surgery [24]. Yet even with chemoradiotherapy, 5-year survival in locally advanced esophageal cancer remains less than $50 \%$ [4]. Only $29 \%$ of patients have a pathologic CR after chemoradiation [4]. Interestingly in our study, a complete CR was observed in a much higher proportion of patients than has been documented in the literature. A local CR was observed in $67 \%$ of patients, and $56 \%$ of patients had a complete CR (no tumor on post-treatment mucosal biopsy or PET scan). These findings raise the possibility that cryotherapy could work synergistically with chemoradiation to increase remission rates. Some investigators have postulated that cryotherapy could stimulate a host antitumor response. Immune responses against malignant cells in the tumor microenvironment are believed to influence prognosis of solid tumors [27]. Tumor infiltrating lymphocytes (TILs) of various subtypes represent the host to tumor reaction. Specific TIL subsets are thought to promote either tumor progression or regression [28]. For instance, in colorectal cancer, CD8+ T cells have been shown to mediate antitumor immunity and to lyse tumors directly by recognizing the major histocompatibility complex class 1 (MHC1) that is present on most tumor cells [28]. In contrast, T-regulatory cells (TREGs) and Forkhead box P3+ (FOXp3+) T cells suppress the immune response of other cells, that is, they affect the inflammatory process indirectly [29]. Given the paucity of data on cryotherapy on the host tumor immune response, our group has recently developed a protocol to compare TILs and immunohistochemistry in pretreatment and post-treatment tumor samples among patients who received cryotherapy and chemoradiation to matched patients who received chemoradiation alone.

This is the first prospective clinical trial of cryotherapy in esophageal cancer, and the first study assessing its safety and usefulness in the definitive or neoadjuvant chemoradiation setting. Our findings need to be interpreted in the context of certain limitations. Nearly all patients were elderly males, which may limit overall generalizability. Given that this was a pilot study without a control group and because six of 21 patients underwent esophageal dilation, the efficacy of cryotherapy at palliating dysphagia may have been overestimated. Although patients were permitted to try solid food as tolerated after 72 hours, they were asked to avoid hard, crunchy foods for a week. These instructions may offer one explanation for why improvement in dysphagia was lower at 2 weeks than at 1 week. Remission rate was a secondary outcome and was assessed based on data from only nine patients, so the high clinical CR rates require further validation. Given the morbidity of esophagectomy, and the advanced age and frailty of our patient population, none of the patients underwent surgery after chemoradiation. As a result, $C R$ was based on mucosal biopsy and PET scan (clinical CR) rather than surgical explant and PET scan (pathologic CR), which could overestimate remission rate [30]. Our general practice is to perform surveillance endoscopy with biopsy every 3 months for the first year after chemoradiation in patients with negative biopsies who do not undergo surgery. During surveillance, one patient had a recurrent tumor within the first year after chemoradiation was completed. If longterm response is considered as a surrogate measure for pathologic CR, then the remission rate would actually be $44 \%$.

\section{Conclusion}

In summary, spray cryotherapy improves dysphagia when administered at initial diagnosis of esophageal cancer. Its use prior to chemoradiation does not appear to increase toxicity. Further study is required to understand whether cryotherapy has any impact on the host tumor immune response $[9,10]$. Our study provides a scientific rationale to pursue larger clinical trials addressing synergistic effects of combining cryotherapy with chemoradiation.

\section{Competing interests}

Dr. Shah received funding from the American Society for Gastrointestinal Endoscopy (ASGE) to conduct the study. Dr. Shah and Dr. Smallfield served on a research advisory board 
for CSA Medical, Inc. Dr. Smallfield receives research support from CSA Medical, Inc.

\section{References}

[1] Ajani JA, D’Amico TA, Almhanna K et al. Esophageal and esophagogastric junction cancers, version 1.2015. J Natl Compr Canc Netw 2015; 13: 194-227

[2] Wang DB, Zhang X, Han HL et al. Neoadjuvant chemoradiotherapy could improve survival outcomes for esophageal carcinoma: a metaanalysis. Dig Dis Sci 2012; 57: 3226-3233

[3] Gkika E, Gauler T, Eberhardt W et al. Long-term results of definitive radiochemotherapy in locally advanced cancers of the cervical esophagus. Dis Esophagus 2014; 27: 678-684

[4] van Hagen P, Hulshof MC, van Lanschot JJ et al. Preoperative chemoradiotherapy for esophageal or junctional cancer. N Engl ] Med 2012; 366: $2074-2084$

[5] Francis SR, Orton A, Thorpe C et al. Toxicity and outcomes in patients with and without esophageal stents in locally advanced esophageal cancer. Int J Radiat Oncol Biol Phys 2017; 99: 884-894

[6] Ohnmacht GA, Allen MS, Cassivi SD et al. Percutaneous endoscopic gastrostomy risks rendering the gastric conduit unusable for esophagectomy. Dis Esophagus 2006; 19: 311-312

[7] Greenwald BD, Dumot JA. Cryotherapy for Barrett's esophagus and esophageal cancer. Curr Opin Gastroenterol 2011; 27: 363-367

[8] Greenwald BD, Dumot JA, Horwhat JD et al. Safety, tolerability, and efficacy of endoscopic low-pressure liquid nitrogen spray cryotherapy in the esophagus. Dis Esophagus 2010; 23: $13-19$

[9] Grana L, Ablin RJ, Goldman S et al. Freezing of the esophagus: histological changes and immunological response. Int Surg 1981; 66: $295-301$

[10] Sabel MS. Cryo-immunology: a review of the literature and proposed mechanisms for stimulatory versus suppressive immune responses. Cryobiology 2009; 58: 1-11

[11] Baust JG, Bischof JC, Jiang-Hughes S et al. Re-purposing cryoablation: a combinatorial 'therapy' for the destruction of tissue. Prostate Cancer Prostatic Dis 2015; 18: 87 - 95

[12] Barthel JS, Kucera S, Harris C et al. Cryoablation of persistent Barrett's epithelium after definitive chemoradiation therapy for esophageal adenocarcinoma. Gastrointest Endosc 2011; 74: 51 - 57

[13] Dumot JA, Vargo JJ, Falk GW et al. An open-label, prospective trial of cryospray ablation for Barrett's esophagus high-grade dysplasia and early esophageal cancer in high-risk patients. Gastrointest Endosc 2009; 70: 635-644

[14] Shaheen NJ, Greenwald BD, Peery AF et al. Safety and efficacy of endoscopic spray cryotherapy for Barrett's esophagus with highgrade dysplasia. Gastrointest Endosc 2010; 71: 680-685

[15] Cash BD, Johnston LR, Johnston MH. Cryospray ablation (CSA) in the palliative treatment of squamous cell carcinoma of the esophagus. World J Surg Oncol 2007; 5: 34
[16] Goetz M, Malek NP, Kanz L et al. Cryorecanalization for in-stent recanalization in the esophagus. Gastroenterology 2014; 146: 1168 1170

[17] Kachaamy T, Prakash R, Kundranda $M$ et al. Liquid nitrogen spray cryotherapy for dysphagia palliation in patients with inoperable esophageal cancer. Gastrointest Endosc 2018; 88: 447- 455

[18] Mellow MH, Pinkas H. Endoscopic therapy for esophageal carcinoma with Nd:YAG laser: prospective evaluation of efficacy, complications, and survival. Gastrointest Endosc 1984; 30: 334-339

[19] Ribeiro A, Bejarano P, Livingstone A et al. Depth of injury caused by liquid nitrogen cryospray: study of human patients undergoing planned esophagectomy. Dig Dis Sci 2014; 59: 1296 - 1301

[20] Cotton PB, Eisen GM, Aabakken L et al. A lexicon for endoscopic adverse events: report of an ASGE workshop. Gastrointest Endosc 2010; 71: $446-454$

[21] Chao YK, Wen YW, Chang HK et al. An analysis of factors affecting the accuracy of endoscopic biopsy after neoadjuvant chemoradiotherapy in patients with esophageal squamous cell carcinoma. Eur J Surg Oncol 2017; 43: $2366-2373$

[22] Kachaamy T, Shah T, Weber J et al. Cryotherapy in combination with systemic chemotherapy for gastro-esophageal cancer palliation: chilling initial results, in Digestive Disease Week 2015. Gastrointestinal Endoscopy AB519 [unpublished abstract]

[23] Divine G, Horton HJ, Hunt R et al. Statistical grand rounds: a review of analysis and sample size calculation considerations for Wilcoxon tests. Anesth Analg 2013; 117: 699-710

[24] Stahl M, Stuschke M, Lehmann N et al. Chemoradiation with and without surgery in patients with locally advanced squamous cell carcinoma of the esophagus. J Clin Oncol 2005; 23: 2310-2317

[25] Yu FJ, Shih HY, Wu CY et al. Enteral nutrition and quality of life in patients undergoing chemoradiotherapy for esophageal carcinoma: a comparison of nasogastric tube, esophageal stent, and ostomy tube feeding. Gastrointest Endosc 2018; 88: 21-31 e4

[26] Lightdale C], Heier SK, Marcon NE et al. Photodynamic therapy with porfimer sodium versus thermal ablation therapy with Nd:YAG laser for palliation of esophageal cancer: a multicenter randomized trial. Gastrointest Endosc 1995; 42: 507 - 512

[27] Grivennikov SI, Greten FR, Karin M. Immunity, inflammation, and cancer. Cell 2010; 140: 883-899

[28] Gooden M], de Bock GH, Leffers $\mathrm{N}$ et al. The prognostic influence of tumour-infiltrating lymphocytes in cancer: a systematic review with meta-analysis. Br J Cancer 2011; 105: 93-103

[29] Zhuo C, Xu Y, Ying M et al. FOXP3+ Tregs: heterogeneous phenotypes and conflicting impacts on survival outcomes in patients with colorectal cancer. Immunol Res 2015; 61: 338-347

[30] Schneider PM, Metzger R, Schaefer $\mathrm{H}$ et al. Response evaluation by endoscopy, rebiopsy, and endoscopic ultrasound does not accurately predict histopathologic regression after neoadjuvant chemoradiation for esophageal cancer. Ann Surg 2008; 248: $902-908$ 